\title{
Ribosomal proteins L11 and L5 activate TAp73 by overcoming MDM2 inhibition
}

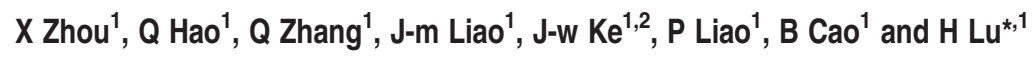

Over the past decade, a number of ribosomal proteins (RPs) have been found to have a role in activating the tumor suppressor p53 by directly binding to MDM2 and impeding its activity toward p53. Herein, we report that RPL5 and RPL11 can also enhance the transcriptional activity of a p53 homolog TAp73, but through a distinct mechanism. Interestingly, even though RPL5 and RPL11 were not shown to bind to p53, they were able to directly associate with the transactivation domain of TAp73 independently of MDM2 in response to RS. This association led to perturbation of the MDM2-TAp73 interaction, consequently preventing MDM2 from its association with TAp73 target gene promoters. Furthermore, ectopic expression of RPL5 or RPL11 markedly induced TAp73 transcriptional activity by antagonizing MDM2 suppression. Conversely, ablation of either of the RPs compromised TAp73 transcriptional activity, as evident by the reduction of p21 and Puma expression, in response to 5-fluorouracil (5-FU). Consistently, overexpression of RPL5 or RPL11 enhanced, but knockdown of either of them hampered, TAp73-mediated apoptosis. Intriguingly, simultaneous knockdown of TAp73 and either of the RPs was required for rescuing the 5-FU-triggered S-phase arrest of p53-null tumor cells. These results demonstrate a novel mechanism underlying the inhibition of tumor cell proliferation and growth by these two RPs via TAp73 activation.

Cell Death and Differentiation (2015) 22, 755-766; doi:10.1038/cdd.2014.167; published online 10 October 2014

Activation of the tumor suppressor p53 leads to cell cycle arrest, apoptosis or senescence, thereby preventing tumorigenesis. ${ }^{1}$ The p53 family member, p73, also plays a role in tumor suppression. ${ }^{2}$ There are several $p 73$ variants, which are categorized into two groups: one with an intact $\mathrm{N}$-terminal transactivation (TA) domain and the other without this domain $(\Delta N)$. The TAp73 isoforms, particularly TAp73 $\beta$, the most active isoform, can transactivate some of the most important p53 target genes, such as p21, Puma, Noxa and $B a x,{ }^{3}$ leading to cell cycle arrest or apoptosis, while the $\Delta N p 73$ isoforms are often inactive or act as dominant negative inhibitors of TAp73. ${ }^{2}$ Consistent with these studies, the isoform-specific deletion of TAp73 results in increased incidence of spontaneous tumors and genomic instability in mice. 4

Owing to the significant structural homology between p53 and TAp73 (29\% at TA domain, 63\% at DNA-binding domain and $38 \%$ at tetramerization domain), ${ }^{5-7}$ these two proteins are subjected to similar posttranslational regulations. For instance, the E3-ubiquitin ligase MDM2, encoded by a gene that can be transactivated by the $p 53$ family, ${ }^{8,9}$ can suppress both p53 and TAp73 activity. MDM2 was originally identified as a p53-interacting protein which associates with both $\mathrm{N}$ - and C-termini of p53 and mediates its ubiquitination and proteasomal degradation. ${ }^{10-13}$ Also, MDM2 can suppress p53 transcriptional activity via their direct binding independently of the E3-ligase activity. ${ }^{14}$ However, previous studies by us and others showed that MDM2 also suppresses TAp73 transcriptional activity ${ }^{15-17}$ by directly binding to the $\mathrm{N}$-terminal TA domain of this transcriptional factor, consequently leading to the inhibition of TAp73-triggered apoptosis without affecting TAp73 stability. ${ }^{15-17}$ Hence, MDM2 is a negative feedback regulator of both $\mathrm{p} 53$ and TAp73.

Over the past decade, the MDM2-p53 feedback loop has been shown to be regulated by a number of ribosomal proteins (RP), including RPL5, ${ }^{18}$ RPL6, $^{19}$ RPL11, ${ }^{20,21}$ RPL23, ${ }^{22,23}$ RPS7, ${ }^{24,25}$ RPS $14^{26}$ and RPS27a ${ }^{27}$ under certain circumstances. Although these RPs are normally utilized for the assembly of the translational machinery-ribosomes essential for protein production, they can individually interact with MDM2 in response to ribosomal stress (RS) or nucleolar stress, and inhibit MDM2-mediated p53 ubiquitination and degradation, leading to p53-dependent cell cycle arrest or growth suppression. ${ }^{28,29}$

The fact that MDM2 interacts with TAp73 and represses its transcriptional activity, as mentioned above, prompted us to determine whether this MDM2-TAp73 feedback loop is also subjected to the regulation by any of these RPs. Indeed, this is the case. Here we report our studies on RPL5 and RPL11. Surprisingly, these two RPs directly bound to the N-terminal TA domain of TAp73 independently of MDM2 upon RS, even though they did not bind to p53. ${ }^{27}$ Consequently, this binding interfered with the MDM2 association with the same domain of TAp73. Consistently, RPL11 and RPL5 impeded MDM2

\footnotetext{
${ }^{1}$ Department of Biochemistry \& Molecular Biology, Tulane Cancer Center; Tulane University School of Medicine; New Orleans, Louisiana, USA and ${ }^{2}$ Department of Laboratory Medicine; Jiangxi Children's Hospital, Nanchang, Jiangxi, China

${ }^{*}$ Corresponding author: H Lu, Department of Biochemistry \& Molecular Biology, Tulane Cancer Center, Tulane University School of Medicine, 1430, New Orleans 70112, Louisiana, USA. Tel: +1 504988 5293; Fax: +1 504988 1611; E-mail: hlu2@tulane.edu

Abbreviations: RP, ribosomal protein; RS, ribosomal stress; TA domain, transactivation domain; $\Delta \mathrm{N}$, deleted N-terminal; 5-FU, 5-fluorouracil; Doxy, doxycycline; IP, immunoprecipitation; IB, immunoblot; ChIP, chromatin immunoprecipitation; LC, light chain; FACS, fluorescence activated cell sorting; RT, Reverse transcription; qPCR, quantitative PCR

Received 23.4.14; revised 09.9.14; accepted 10.9.14; Edited by KH Vousden; published online 10.10.14
} 
association with TAp73 target gene promoters, and therefore, bolstered the TAp73 transcriptional activity and induced TAp73-dependent apoptosis. Inversely, siRNA-mediated ablation of these RPs attenuated TAp73 activity and alleviated p73-dependent apoptosis and cell cycle arrest. This study, as detailed below, unveils RPL5 and RPL11 as new positive regulators of TAp73 by circumventing MDM2 inhibition.

\section{Results}

RPL11 and RPL5 bind to N-terminal domain of TAp73. Previously, we and others showed that RPL5, RPL11 and RPS14 act as p53 activators by abrogating MDM2 E3ubiquitin ligase activity. ${ }^{18,20,21,26}$ As MDM2 also negates TAp73 transcriptional activity, ${ }^{15-17}$ we determined whether any of these RPs might regulate the TAp73 activity by overcoming the MDM2 negation. First, we tested whether they can bind to TAp73 in cells by conducting a set of coimmunoprecipitation (IP)-immunoblot (IB) assays after transfecting p53-null human lung cancer H1299 cells with a Flagtagged plasmid that expresses RPL5, RPL11, RPL30, RPS12, RPS14, RPS19 or RPS27a, together with the TAp73 plasmid. As shown in Figure 1a, TAp73 was co-immunoprecipitated with RPL5, RPL11 and RPS14, respectively, whereas it hardly associated with any of RPL30, RPS12, RPS19 and RPS27a (Figure 1a). We focused on RPL5 and RPL11, in this study, as they can regulate the MDM2-p53 loop in both in vitro and in vivo model systems. ${ }^{30-33}$ The interaction of RPL5 or RPL11 with TAp73 was further verified using an anti-GFP antibody for the inverse co-IP assay (Figure 1b). Surprisingly, the RPL5- or RPL11-TAp73 interaction was MDM2-independent as ectopic TAp73 was also co-immunoprecipitated with ectopic RPL5 or RPL11 in MEF ${ }^{\mathrm{MDM} 2-/-/ \mathrm{p} 53-/-}$ cells (Figure 1c). However, similar to MDM2, ${ }^{15}$ RPL5 and RPL11 required the N-terminal domain of TAp73 for their interactions as well, as either of them was unable to bind to $\Delta \mathrm{Np} 73$ (Figures $1 \mathrm{~d}$ and $1 \mathrm{e}$ ). To further determine whether these RPs might bind to p53 in the tested cells, we also conducted co-IP-IB assays as shown in Figure $1 f$ and found that RPL5 and RPL11 again bound to TAp73, but not p53 (Figure 1f). These results indicate that RPL5 and RPL11 can directly bind to the N-terminal domain of TAp73.

RPL5 and RPL11 bind to TAp73 upon RS. Next, we wanted to determine if the interaction of RPL5 and RPL11 with TAp73 occurs upon RS. We previously showed that 5-FU treatment induces the interaction of RPL5 and RPL11 with MDM2 and consequently induces p53 and p53-mediated cell cycle a
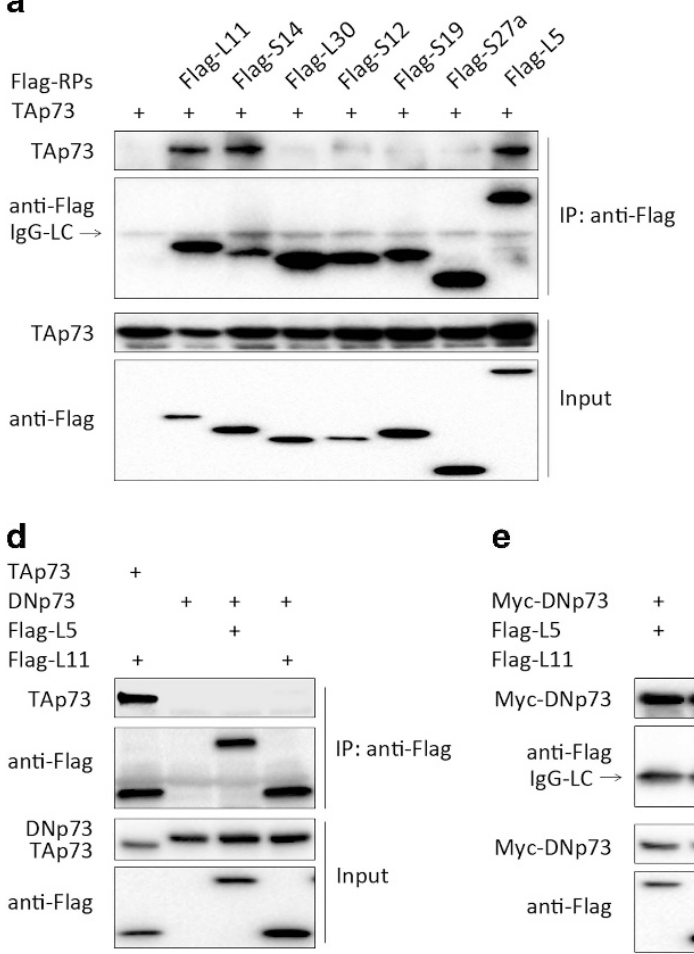

b

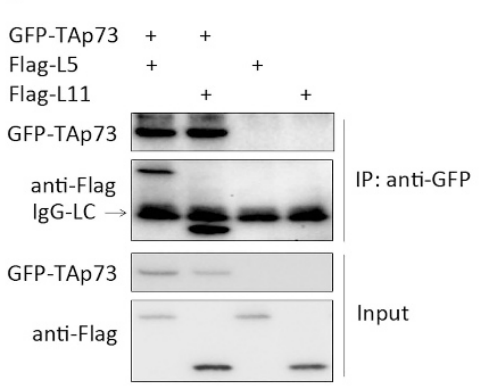

C

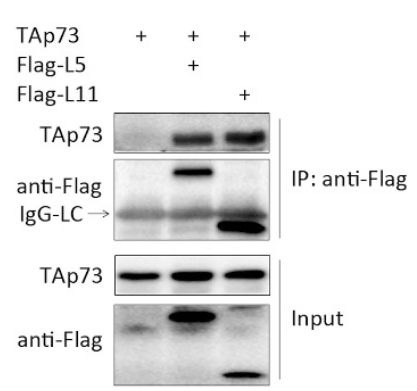

e

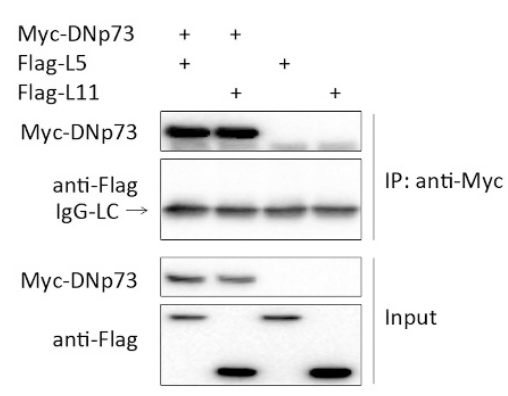

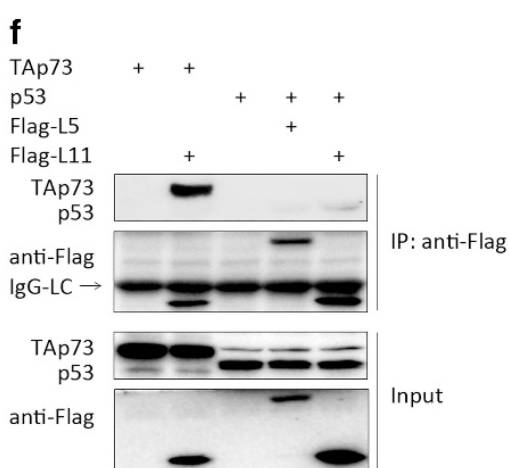

Figure 1 RPL5 and RPL11 associate with TAp73 independently of MDM2. (a) Exogenous RPL5, RPL11 and RPS14 bind to exogenous TAp73. H1299 cells were transfected with combinations of Flag-tagged RPs as indicated together with non-tagged TAp73 expression plasmids for $30 \mathrm{~h}$ and harvested for IP assay using an anti-Flag antibody, followed by IB analyses using the antibodies as indicated. (b) Exogenous RPL5 and RPL11 bind to GFP-TAp73. The same experiments were conducted as shown in (a) except for using the GFP-tagged TAp73 expression plasmid for transfection and anti-GFP for IP assays. (c) RPL5 and RPL11 bind to TAp73 independently of MDM2. The same experiments were

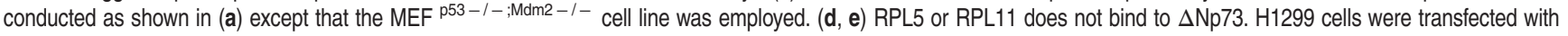
combinations of Myc- $\Delta$ Np73 and Flag-tagged RPs as indicated followed by reciprocal IP assays using anti-Flag (d) or anti-Myc (e) antibodies. (f) RPL5 or RPL11 does not bind to p53. H1299 cells were transfected with combinations of V5-p53 and Flag-tagged RPs as indicated followed by IP assays using anti-Flag 
arrest, ${ }^{31}$ yet it has not been shown that 5 -FU could really cause RS by interrupting ribosome biogenesis and releasing ribosome-free RPs. To do so, we treated HCT116 ${ }^{\mathrm{p} 53-1-}$ colon-cancer cells with 5 -FU followed by ribosome profiling analysis. Under a normal condition, most RPs were associated with monosomes (including 40S, 605 and $80 S$ ) and polysomes, while RPs could be hardly detectable in the free-form fractions (Figure 2a). By striking contrast, in the 5-FU-treated samples, ribosome biogenesis was severely impaired as evident by the reduction of 805 assembly, but accumulation of ribosome-free RPs (Figure $2 b$ ). Given that TAp73 was only found in the free-form fractions rather than ribosomeassociated fractions (Figures $2 a$ and $b$ ), these results suggest that 5-FU-induced RS might enhance the interaction of endogenous RPL5 and RPL11 with TAp73. Indeed, when 5-FU-treated and ribosome-free fractions were used for co-IP-IB assays (Figure 2b), TAp73 was co-pulled down with RPL5 and RPL11 (Figure 2c).
In addition, we employed a TAp73-inducible osteosarcoma Saos2 cell line for testing RP-TAp73 interaction and for the following experiments. In the cells, the expression of HA-TAp73 is induced by Doxycycline (Doxy) in a dose- or time-dependent manner (Supplementary Figure S1). ${ }^{34}$ The cells were treated with Doxy and 5-FU, and used for co-IP assays. As shown in Figure 2d, endogenous RPL5 and RPL11 were co-immunoprecipitated with the induced TAp73 by an anti-HA, but not IgG, antibody. When cells were treated with or without Doxy, the endogenous RPL5 and RPL11 were co-immunoprecipitated with TAp73, whereas this was not the case in the absence of TAp73 (Figure 2e). Also, we have detected the endogenous binding of RPS14, but not RPL30, to TAp73, consistent with the result of Figure 1a. Altogether, our results demonstrate that endogenous RPL5 and RPL11 can bind to TAp73 in response to $\mathrm{RS}$. a
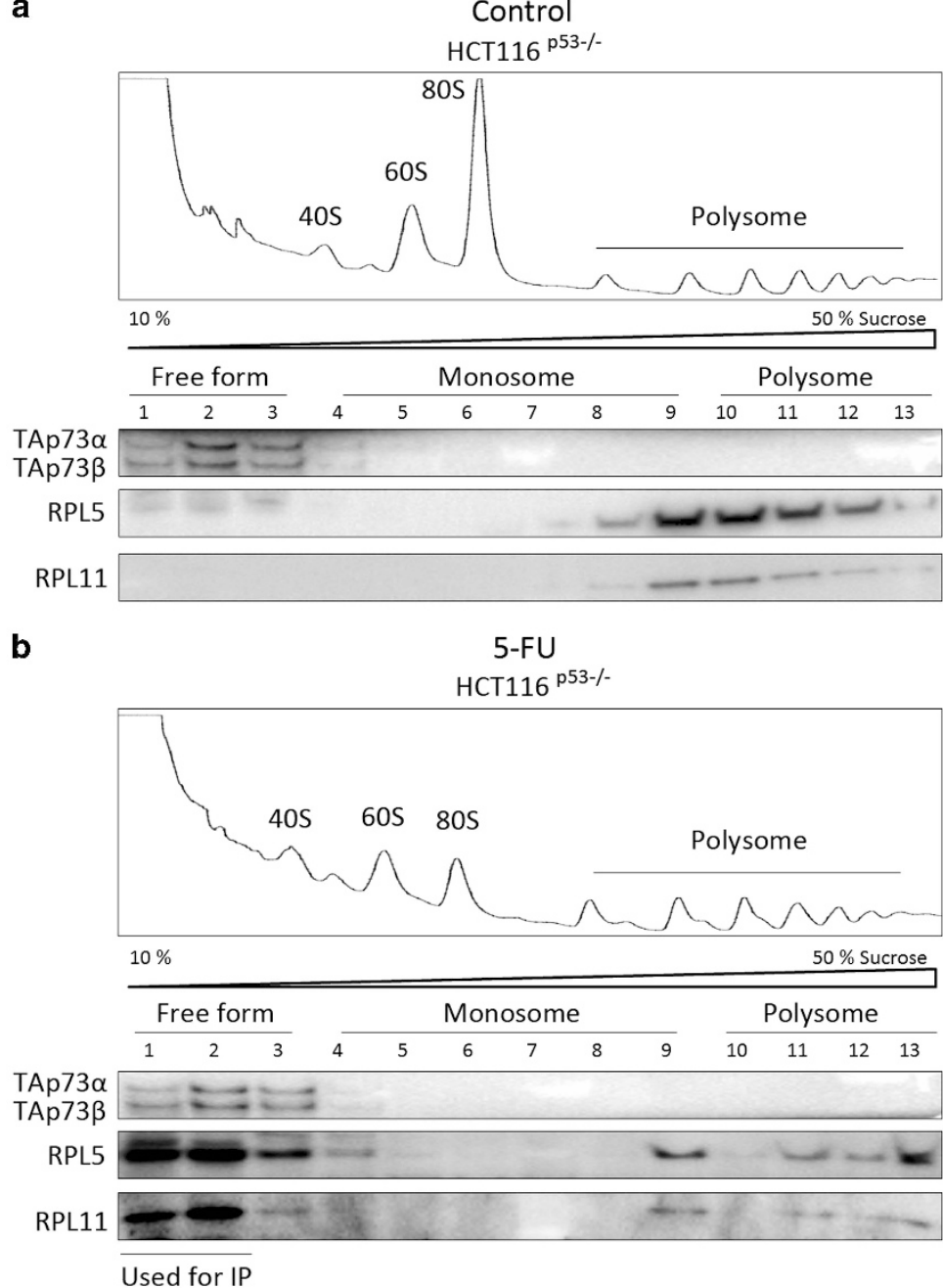

c

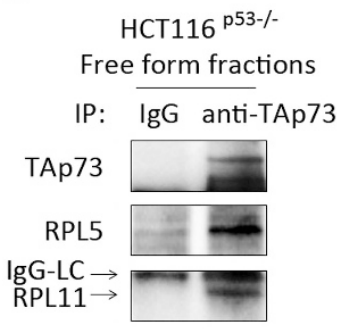

d TAp73-inducible Saos2

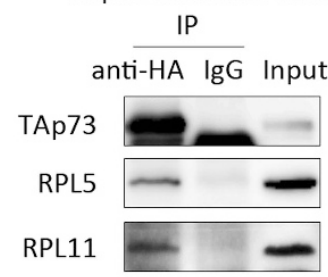

e

TAp73-inducible Saos2

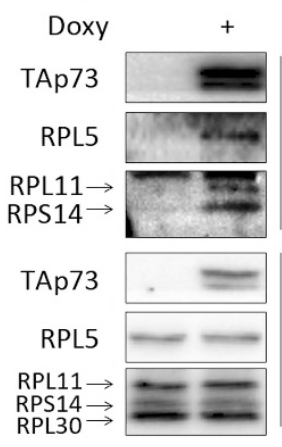

IP: anti-HA beads

nput

Figure 2 Nucleolar stress triggers endogenous RP and TAp73 binding. (a, b) Ribosome profiles of cells untreated (a) or treated (b) with 5-FU show that nucleolar stress causes accumulation of RPs in the free-form fractions. (c) Endogenous RPL5 and RPL11 associate with endogenous TAp73 in the free-form factions upon 5-FU treatment. (d, e) Endogenous RPL5 and RPL11 bind to TAp73 in the Saos2 cells. The HA-tagged TAp73-inducible Saos2 cells were treated with 5-FU and Doxycycline followed by IP assays with anti-HA antibody or IgG (d). Saos2 cells with or without induced TAp73 were subjected to IP assays using anti-HA conjugated agarose beads (e). The co-immunoprecipited complex was detected by IB using antibodies as indicated 
RPL5 and RPL11 perturb MDM2-TAp73 interaction without engaging MDM2-RP binding. As both MDM2 and RPL5/RPL11 associate with the TA domain of $p 73$, it is possible that these RPs might influence the MDM2-TAp73 interaction. To test this idea, we conducted a set of co-IP-IB assays after transfecting $\mathrm{H} 1299$ cells with combinations of different protein-expression plasmids as indicated in Figure 3a. As expected, ${ }^{15}$ MDM2 bound to TAp73, but remarkably, overexpression of RPL11 or RPL5 reduced the formation of the MDM2-TAp73 complex in cells (Figure 3a). Because both RPs bind to MDM2 as well, ${ }^{18,20,21}$ we then tested whether this reduction requires the RP-MDM2 binding by employing an in vitro GST protein-protein interaction assay with purified GST-TAp73, His-RPs and mutant MDM2 (C308W) proteins. This MDM2-C308W mutant is defective in binding to RPL5 and RPL11 as shown in Supplementary
Figure S2A and previously described. ${ }^{35}$ We showed in Figure $3 b$ that GST-TAp73, but not GST, could pull down His-MDM2-C308W in vitro. His-RPL5 or His-RPL11 not only bound to GST-TAp73 directly in vitro, but also interfered with the interaction between MDM2-C308W and TAp73 in a dose-dependent manner (Figure $3 b$ ). These results reveal that the association of either of the RPs with the TA domain of TAp73 can perturb the MDM2-TAp73 interaction, which is independent of their MDM2 binding.

RPL5 and RPL11 prevent MDM2 from binding to TAp73 at target gene promoters. Although MDM2 suppresses TAp73 activity, ${ }^{15-17}$ it remains unknown if this action is through the promoter association of MDM2 with TAp73 and if RPL5 and/or RPL11 can disrupt this association. To address these questions, we performed a series of a

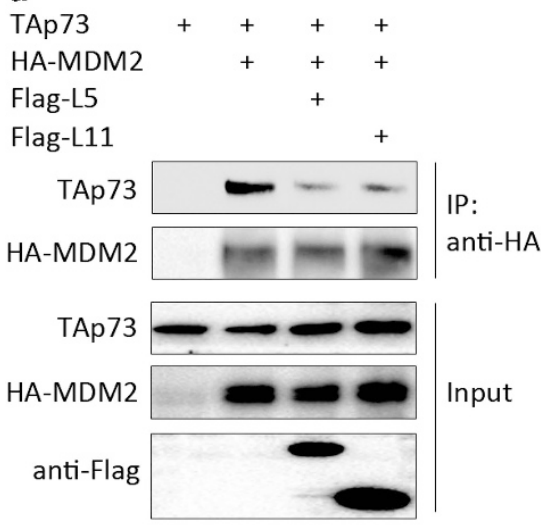

b

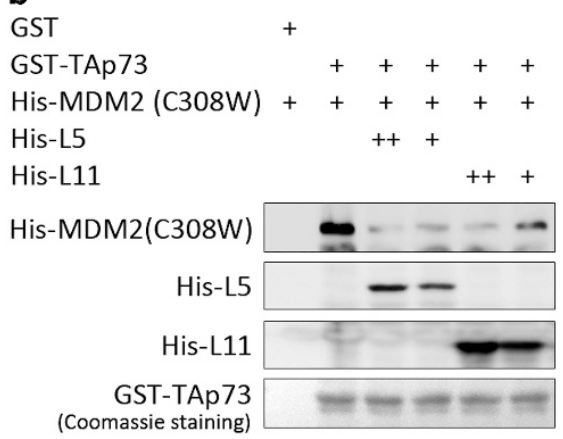

c
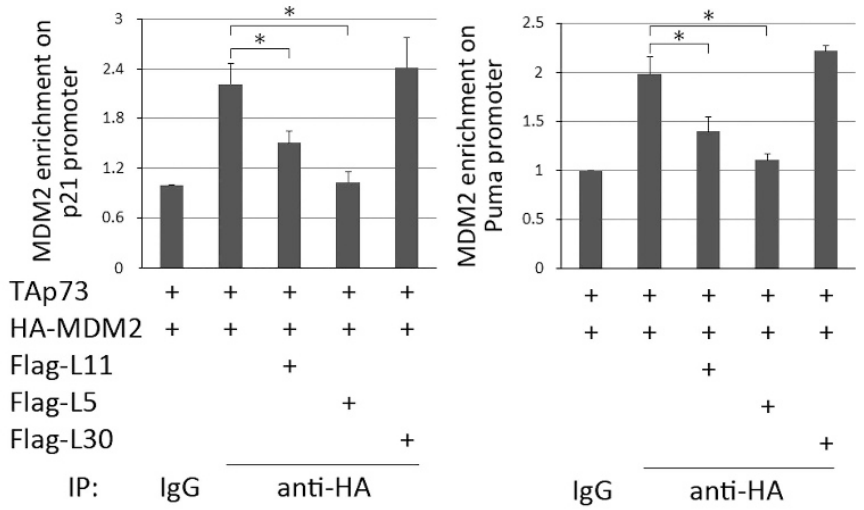

d

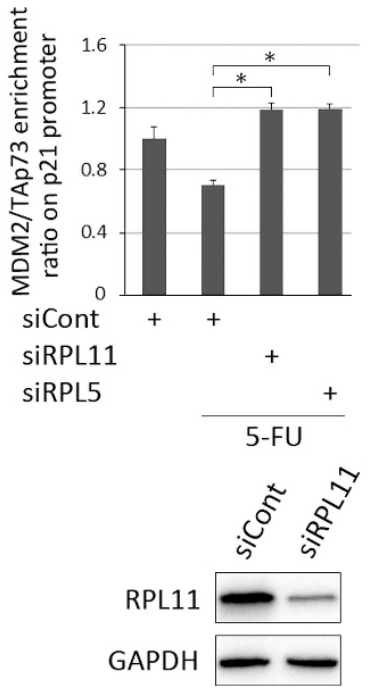

RPL5

GAPDH

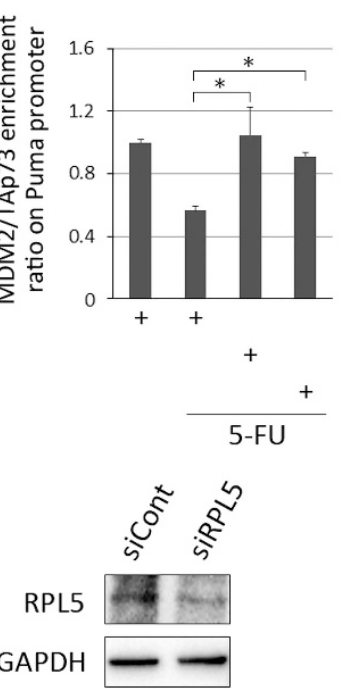

Figure 3 RPL5 and RPL11 perturb MDM2-TAp73 interaction, thus hampering MDM2 recruitment on the TAp73 target gene promoters. (a) Perturbation of MDM2-TAp73 interaction. H1299 cells were transfected with combinations of TAp73, HA-MDM2, Flag-RPL5 and Flag-RPL11 expression plasmids for $30 \mathrm{~h}$ and harvested for IP assays using anti-HA, followed by IB analyses using antibodies as indicated. (b) The perturbation of MDM2-TAp73 interaction does not require the binding of RPs and MDM2. GST-pull down assay was performed using the indicated bacterial expressed proteins, followed by IB analyses using antibodies as indicated. (c) RPL5 and RPL11, but not RPL30, reduce the MDM2 association with TAp73 target gene promoters. H1299 cells were transfected with indicated plasmids and subjected to ChIP assays using anti-HA or mouse lgG, followed by qPCR analyses of the p53/p73 responsive elements on the $p 21$ and Puma promoters. (*) $P$-value $\leq 0.05$. (d) Ablation of RPL5 or RPL11 increases the MDM2/TAp73 occupancy ratio on $p 21$ and Puma promoters. HCT116 $6^{\mathrm{p} 3-l-}$ cells were transfected with siRNAs against RPL5, RPL11 or control for $48-72 \mathrm{~h}$ and treated with $5-\mathrm{FU}$ for $15 \mathrm{~h}$ before harvesting, followed by ChIP assays using anti-MDM2 and anti-p73. $\left(^{*}\right) P$-value $\leq 0.05$ 
chromatin-immunoprecipitation (ChIP) assays. Indeed, MDM2 was enriched on the promoters of TAp73 target genes, such as p21 and Puma, by approximately twofolds, and this increment was markedly reduced by ectopic RPL5 or RPL11, but not RPL30 (Figure 3c). This effect was not due to the change of MDM2 or TAp73 protein levels (Supplementary Figure S2B). Next, we tested if endogenous RPs can regulate the association of MDM2 with TAp73 target promoters. Because the relative ratio of p53 to MDM2 is critical for p53 function, and RPL11 was shown to alleviate the ratio of MDM2 occupancy/p53 occupancy on the p21 promoter in response to $\mathrm{RS}^{36}$ we compared the MDM2/TAp73 occupancy ratio on the $\mathrm{p} 21$ and Puma promoters in HCT116 ${ }^{\mathrm{p} 53-/-}$ cells with or without 5 -FU treatment by conducting ChIP assays. Interestingly, the MDM2/TAp73 ratio on the target gene promoters was reduced in response to 5-FU treatment (Figure 3d), which triggers RS (Figures $2 a$ and b). ${ }^{31}$ This reduction was rescued by further knocking down either RPL5 or RPL11 (Figure 3d), indicating that the reduction of MDM2 occupancy on TAp73 target promoters by $5-\mathrm{FU}$ is due to the dissociation of the MDM2-TAp73 complex at the promoters by these RPs. As all experiments were performed in the p53-null background, to test whether these RPs regulate MDM2TAp73 cascade in the presence of p53, we also conducted some experiments in HCT116 ${ }^{\mathrm{p} 53+/+}$ cells (Supplementary Figures S2C and S2D). We found that RPL5 and RPL11 still bound to TAp73 (Supplementary Figure S2C) and reduced MDM2 recruitment on TAp73/p53 target gene promoters (Supplementary Figure S2D), indicating that these RPs might also regulate TAp73 activity regardless of the presence of p53 or not. Taken together, these results demonstrate that RPL5 and RPL11 can prevent the association of MDM2 with TAp73 on TAp73's target gene promoters.

\section{RPL5 and RPL11 relieve feedback suppression of TAp73} by MDM2. To determine the functional outcome of the interruption of MDM2-TAp73 binding by these RPs, we first performed luciferase reporter assays by transfecting $\mathrm{H} 1299$ cells with combinations of plasmids encoding TAp73, HAMDM2, Flag-tagged RPL5, RPL11 or RPL30 together with a pGL4-miR-34a plasmid containing a p53-responsive element. As expected, TAp73 drastically induced the luciferase activity, which was enhanced by RPL5 and RPL11, but was suppressed by MDM2. Importantly, RPL5 and RPL11, rather than RPL30, also alleviated MDM2-mediated inhibition of luciferase activity (Figure 4a). In addition, RPL5 and RPL11 rescued the expression of endogenous p21 and Puma mRNAs from the inhibition of TAp73 activity by MDM2 (Figures $4 b$ and $c$ ). Together, these results show that RPL5 and RPL11 can induce TAp73 transcriptional activity by impeding MDM2-mediated suppression.

To further consolidate the above notion, we employed TAp73-inducible Saos2 cells. In the absence of Doxy, the expression of TAp73 and MDM2 was undetectable in this Saos2 cell line (Supplementary Figure S1), and ectopic RPs barely affected the expression of p21 and Puma as determined by $q P C R$ and IB (Figures $4 d-f$ ). However, in the presence of Doxy, the expression of TAp73 as well as its target gene MDM2 was elevated in a time-dependent manner (Supplementary Figure S1), and furthermore, ectopic RPL5 or RPL11 markedly boosted the mRNA and protein expression of Puma and p21 (Figures 4d-f). Again, these results demonstrate that RPL5 and RPL11, but not RPL30 that was unable to bind to TAp73 (Figure 1a), can indeed enhance TAp73 activity and induce the TAp73-dependent expression of Puma and p21 (Figure 4f). Together with the above data (Figures $1 \mathrm{a}, 2 \mathrm{e}, 3 \mathrm{c}$ and $4 \mathrm{a}$ ), these results demonstrate that RPL5 and RPL11, but not RPL30, specifically enhance TAp73 transcriptional activity.

Endogenous RPL5 and RPL11 are required for TAp73 activity. To ensure that TAp73 activity is regulated by endogenous RPs, we conducted siRNA-mediated ablation in $\mathrm{HCT} 116^{\mathrm{p} 53-1-}$ cells. Consistent with the results in Figure 3d, 5-FU-treatment induced p21 and Puma mRNA levels, and this induction was significantly reversed by depleting RPL5 or RPL11 to different degrees (Figures 5a and b). However, when we examined the protein expression of p21 and Puma after 5-FU treatment, a differential outcome was observed: $5-\mathrm{FU}$ induced the protein level of Puma as well as TAp73 $a$ and TAp73 $\beta$, but reduced the protein level of p21 (Figure 5c). The reduction of p21 protein level could be due to the ubiquitin-mediated degradation by $\mathrm{CRL} 4^{\mathrm{Cdt} 2}{ }^{37}$ or Skp2 ${ }^{38}$ upon DNA damage stress that can be also induced by $5-\mathrm{FU}$. Additionally, several studies also found that p21 can be degraded by $M D M 2^{39,40}$ or through the REGy-proteasome pathway ${ }^{41,42}$ in an ubiquitination-independent manner. ${ }^{43-45}$ Regardless of this difference, ablation of any of these RPs, including RPS14, led to the reduction of both p21 and Puma protein levels in response to 5-FU treatment (Figure $5 \mathrm{c}$ ). The representative knockdown efficiency of these RPs in HCT116 ${ }^{\text {p53-/ - }}$ cells is shown in the bottom panels of Figure 5c. 5-FU may induce TAp73 activity through two mechanisms: by triggering RS, releasing RPs that counteract the negative regulation of TAp73 by MDM2; alternatively, by causing DNA damage stress, resulting in TAp73 stabilization. Our study shows the important role of RPL5 and RPL11 in maintaining TAp73 transcriptional activity, as ablation of any of these two RPs represses the expression of TAp73 target genes despite the elevated TAp73 expression upon 5-FU stimulation (Figures $5 a-c$ ). This result was further confirmed by using TAp73-inducible Saos2 cells, as knockdown of each of the RPs reduced TAp73-mediated induction of p21 and Puma in the cells (Figure $5 \mathrm{~d}$ ). This result was repeated by examining Puma expression when vector-based shRNAs were used to knockdown RPL5 or RPL11 in HCT116 ${ }^{\text {p53-I- }}$ cells (Supplementary Figure S3).

Next, we tested whether or not the reduction of TAp73 target gene expression is TAp73 or MDM2 dependent. By assessing the expression of TAp73 target genes, we found that the expression of both Puma and Bax was drastically reduced by RPL5 or RPL11 ablation, and this reduction was abrogated by further knocking down TAp73 (Figure 5e). Additionally, MDM2 knockdown was also found to alleviate the deregulation of Puma and Bax caused by RP depletion (Figure 5f). These results indicate that RPL5 and RPL11 do regulate gene expression through the MDM2-TAp73 cascade.

RPL5 and RPL11 promote TAp73-mediated apoptosis and retard cell proliferation. Given that TAp73 was initially 


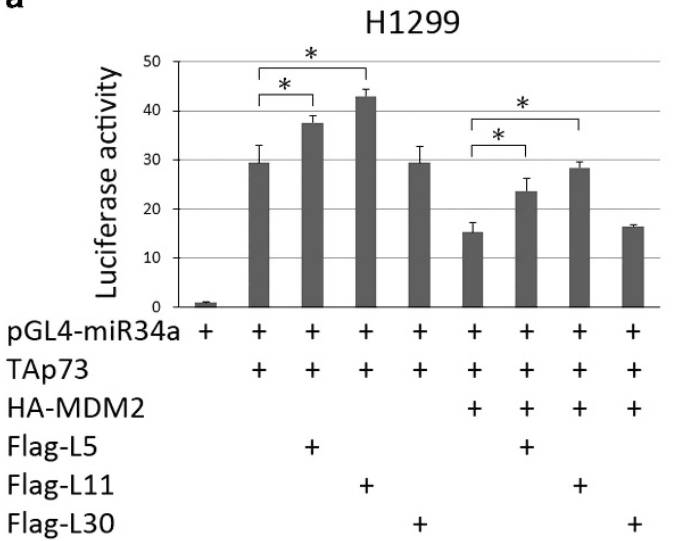

b

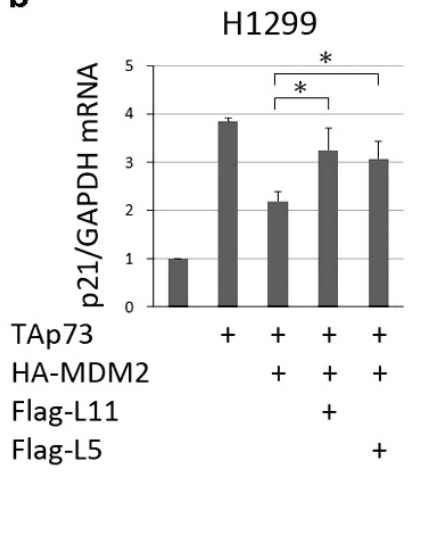

C

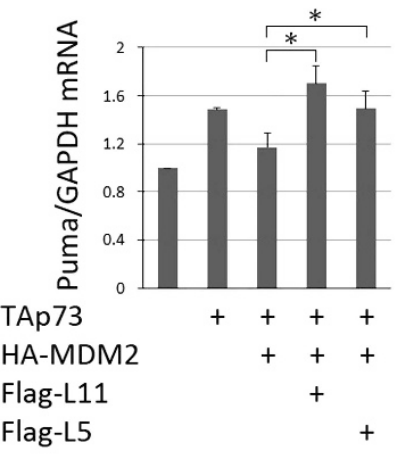

H1299

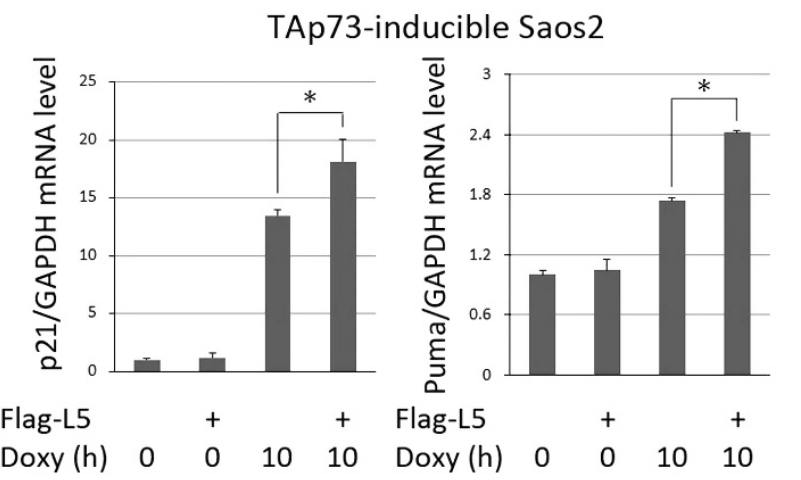

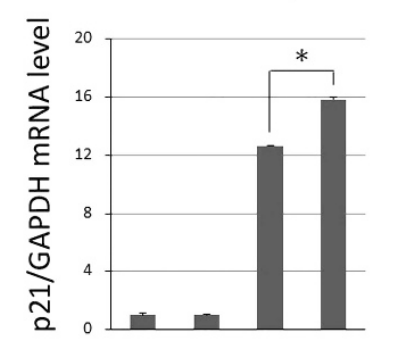

$\begin{array}{lllll}\text { Flag-L11 } & & + & & + \\ \text { Doxy (h) } & 0 & 0 & 10 & 10\end{array}$ f

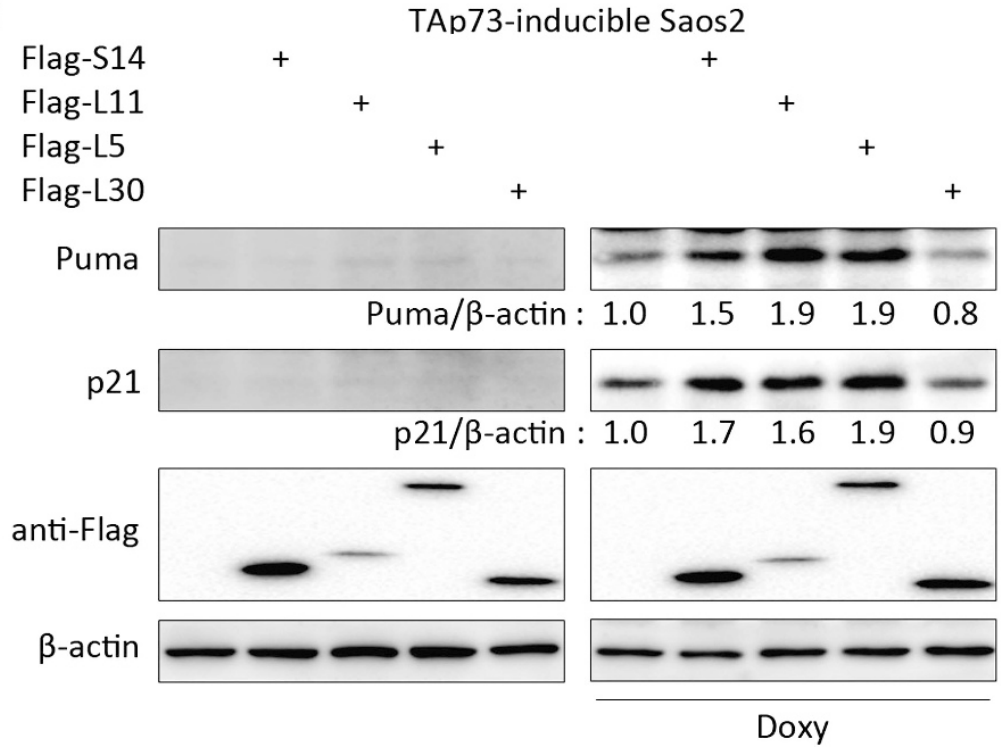

Figure 4 RPL5 and RPL11 induce TAp73 transcriptional activity by inhibiting MDM2. (a) RPL5 and RPL11, but not RPL30, induce TAp73 transcriptional activity in the presence and absence of MDM2. $\mathrm{H} 1299$ cells were transfected with the indicated plasmids together with $\mathrm{pCMV}$ - $\beta$-galactoside as an internal control and harvested for luciferase assays and $\beta$-gal activity determination. (*) $P$-value $\leq 0.05$. (b, c) H1299 cells were transfected with the indicated plasmids for $30 \mathrm{~h}$ and subjected to RT-qPCR analyses of $p 21$ (b) and Puma (c) expression. (*) P-value $\leq 0.05$. (d, e) RPL5 and RPL11 enhance TAp73 activity determined by p21 and Puma mRNA expression. The TAp73-inducible Saos2 cells were transfected with Flag-RPL5 (d) or RPL11 (e) as indicated for over $30 \mathrm{~h}$ and Doxycycline was added $10 \mathrm{~h}$ before harvesting for RT-qPCR analyses. $\left({ }^{*}\right) P$-value $\leq 0.05$. (f) RPL5, RPL11 and RPS14, but not RPL30, enhance TAp73 activity determined by p21 and Puma protein expression. TAp73-inducible Saos2 cells were transfected with Flag-RPs, as indicated, for $48 \mathrm{~h}$ and Doxycycline was added $15 \mathrm{~h}$ before harvesting for IB analyses using the antibodies as indicated 
identified as a tumor suppressor due to its apoptotic property, $^{7}$ and its target genes, Puma and Bax, were shown to be regulated by RPL5 and RPL11, we sought to investigate whether these two RPs promote TAp73-mediated apoptosis. To do so, we introduced ectopic RPL5 or RPL11 into TAp73inducible Saos2 cells and performed fluorescence-activated cell sorting (FACS) analyses. Overexpression of RPL5 or RPL11 without TAp73 induction marginally affected Sub-G1 population, but upon TAp73 induction by Doxy, RPL5 and RPL11 markedly induced the Sub-G1 population (Figures $6 a$ and b). Conversely, ablation of RPL5 or RPL11 significantly impaired TAp73-induced Sub-G1 accumulation, but only showed marginal effect on this population in the absence of TAp73 induction (Figures 6c and d). Also, ectopic RPL5 or RPL11 in the cells without TAp73 induction moderately decreased cell viability compared to the vector control probably due to inactivation of c-Myc. ${ }^{46}$ However, when TAp73 was induced by Doxy, cell viability was more drastically reduced by these two RPs than that of the vector transfected cells (Supplementary Figure S4). Taken together, these results demonstrate that RPL5 and RPL11 can enhance TAp73-dependent apoptosis and cell growth arrest.

RPL5 and RPL11 synergize with TAp73 to elicit S-phase arrest in response to 5-FU. Our group previously found that 5-FU treatment results in accumulation of cells at G1 or early $S$ phase and partial loss of $\mathrm{G} 2$ cells by activating wild-type p53 in human osteosarcoma cell line U2OS. ${ }^{31}$ Here, we wanted to determine whether 5-FU could also cause defects in cell cycle progression in p53-negative cancer cells. Human colon-cancer HCT116 ${ }^{\mathrm{p} 53-/-}$ cells were treated with or without 5-FU for $20 \mathrm{~h}$ and subjected to FACS analyses. Interestingly, 5-FU specifically led to S-phase arrest and complete loss of G2 population in the p53-deficient cancer cells (Figure 7a). However, ablation of TAp73 partially alleviated the effect of 5-FU on the cell cycle arrest by allowing a small portion of S-phase arrested cells to enter G2 phase, indicating that TAp73 contributes to 5-FU-triggered S-phase accumulation (Figure 7a). Remarkably, when we knocked down both RPL5 and TAp73, RPL11 and TAp73, or RPS14 and TAp73, a synergistic effect on the cell cycle was observed (Figure 7a). Particularly, simultaneous depletion of RPL5 and TAp73 in the 5-FU treated cells could drastically restore the cell cycle progression to normal as compared to the untreated cells (Figure 7a). Moreover, we also assessed apoptosis in these cells treated with 5 -FU up to $48 \mathrm{~h}$ by knocking down each RP and found that both RPL5 and RPL11 were required for 5-FU-induced apoptosis (Figure 7b), which is consistent with the aforementioned results shown in Figures 5c,6c and d. Taken together, these results demonstrate that the RPs are required for 5-FU-induced S-phase cell cycle arrest and apoptosis in these p53-deficient cancer cells.

\section{Discussion}

Here, we present a novel mechanism underlying the regulation of TAp73 transcriptional activity by RPs (Figure 7c). By screening a number of RPs for their ability to associate with TAp73, we identified RPL5 and RPL11 as new regulators of this transcriptional activator. Surprisingly, they directly associated with TAp73, but neither $\Delta$ Np73 (Figure 1), nor p53 directly (Figure 1f). ${ }^{18,20,21}$ 5-FU perturbed ribosome assembly and caused accumulation of ribosome-free RPs, thus enhancing the binding of RPL5 and RPL11 to TAp73 (Figure 2). Remarkably, their association with the TA domain of p73 disrupted the MDM2-TAp73 interaction, leading to the disassociation of MDM2 from the TAp73 target gene promoters (Figure 3). Ectopic RPL5 or RPL11 reversed MDM2mediated suppression of TAp73 transcriptional activity (Figure 4), while knockdown of RPL5 or RPL11 impaired TAp73 transcriptional activity (Figure 5). Further analyses showed that RPL5 and RPL11 prompt TAp73-triggered apoptosis and reduce cell proliferation, whereas ablation of RPL5 or RPL11 alleviates TAp73-triggered apoptosis (Figure 6). Intriguingly, RPL5 and RPL11 were also found to be essential for 5-FU-induced S-phase arrest and apoptosis (Figure 7). Of note, RPS14 can also activate TAp73 via a similar mechanism, though this is not the focus of this study.

Our findings together with previous studies by us and others ${ }^{18,20,21}$ demonstrate that RPL5 and RPL11 can activate not only p53, but also p73. However, their mechanisms are different: these two RPs activate p53 by directly binding to the central domain of MDM2 and inhibiting its E3-ubiquitin ligase activity toward p53, consequently stabilizing p53, but they activate $\mathrm{p} 73$ by directly binding to the $\mathrm{N}$-terminus of TAp73 and thus competing with MDM2 for binding to the same domain, consequently circumventing the inhibitory effect of MDM2.

Although it has been shown that several RPs execute their cellular functions by triggering cell cycle arrest at G1 and/or $\mathrm{G} 2$, rather than inducing apoptosis, by activating $p 53,{ }^{28,29}$ to our surprise, RPL5 and RPL11 can actually promote apoptosis in a TAp73-dependent manner in the absence of p53 (Figure 6). This is probably because the expression of apoptosis-associated target genes (Puma and Bax) of TAp73 is more sensitive to the deregulation of RPs, as compared to the p21 expression (Figures 5a, b, e and f). We also observed that RP knockdown could reduce Puma, but not p21, expression even without 5-FU stimulation (Figures $5 \mathrm{a}-\mathrm{c}$ ). This observation suggests that under normal cell culture conditions, there are some basal levels of RS that could provoke the release of some ribosome-free RPs as shown in Figure $2 \mathrm{a}$, and these RPs act to induce TAp73-dependent expression of pro-apoptotic genes, rather than cell cycle genes (e.g., p21), consequently leading to cell apoptosis, as depletion of TAp73 in cells did not cause further reduction of Puma and Bax due to RP knockdown (Figure 5e); also, overexpressing or knocking down RP barely affected apoptosis in Saos2 cells without TAp73 induction (Figures 6a-d). RP-mediated S-phase arrest upon 5-FU treatment seems not through the RP-MDM2-TAp73 cascade, but this requires the cooperation of RPs and TAp73, because ablation of both RPs and TAp73 more dramatically promoted the cell cycle progression compared to that for single knockdown of each gene.

There are other regulators that have also been shown to interact with the TA domain of p73, including MDMX, ${ }^{47,48} \mathrm{p300}$ and Chk $1,{ }^{50}$ which may affect the stability, transcriptional activity or phosphorylation status of TAp73. It would be worthwhile to determine whether the RPs can regulate TAp73 activity by interplaying with these proteins as well in 

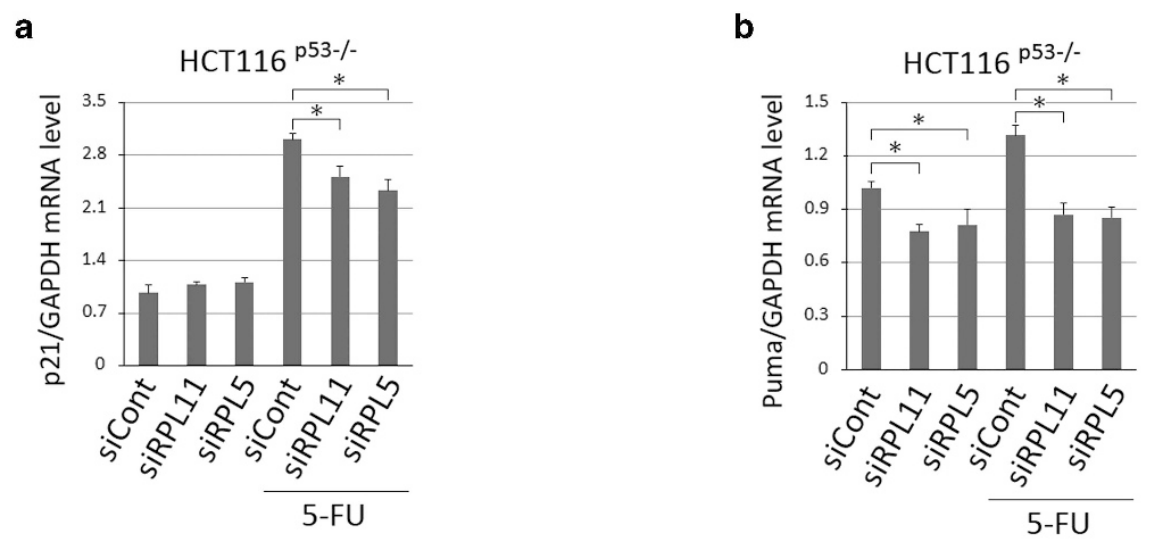

c

HCT116 ${ }^{\mathrm{p53-1-}}$

d
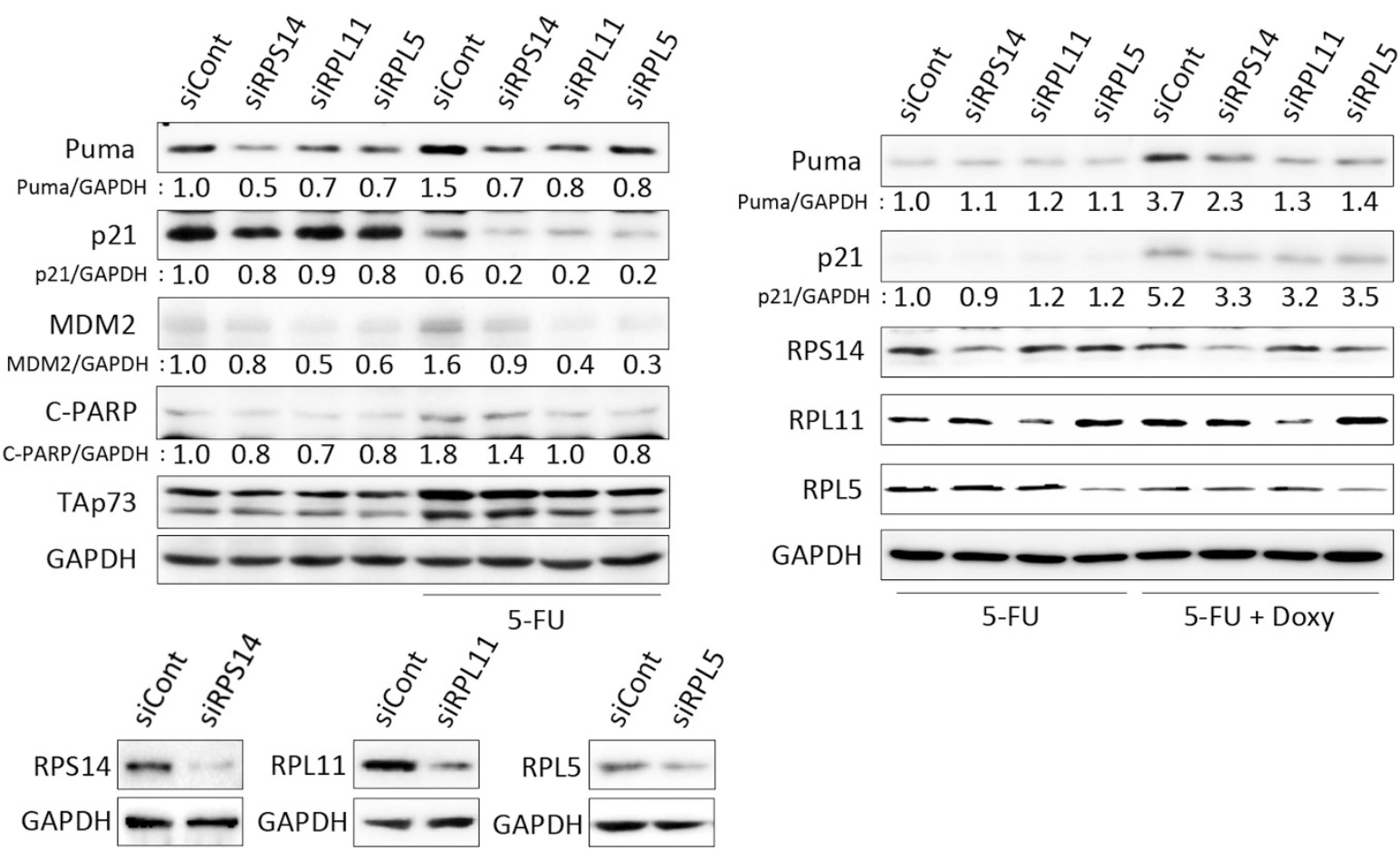

e
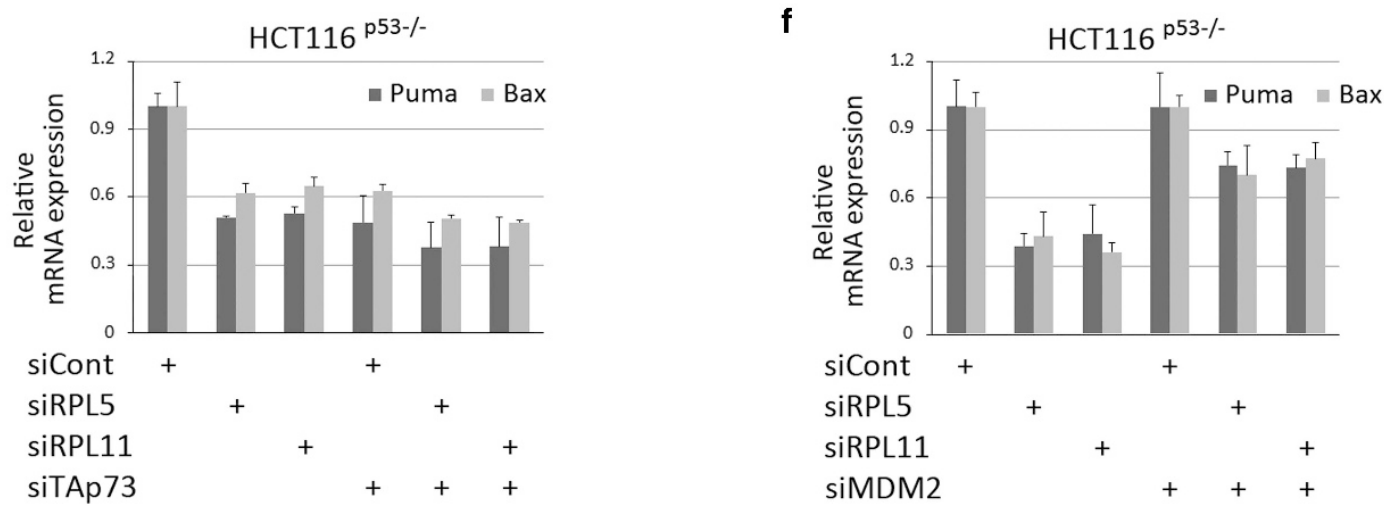
a
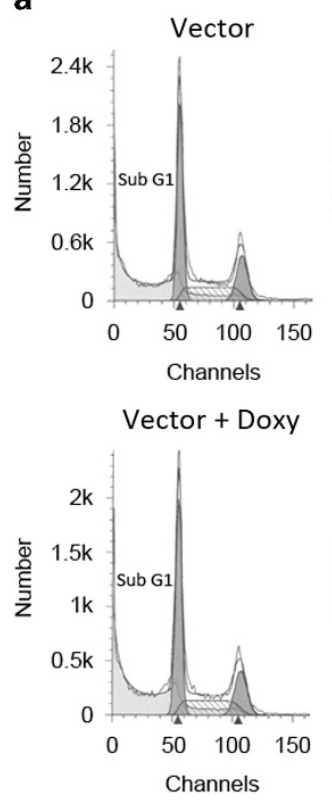

c

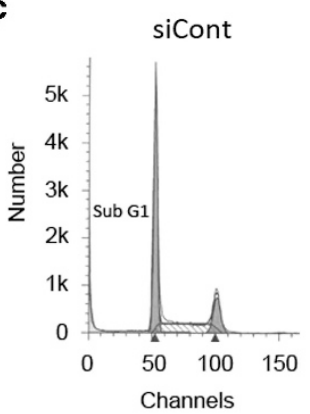

siCont + Doxy

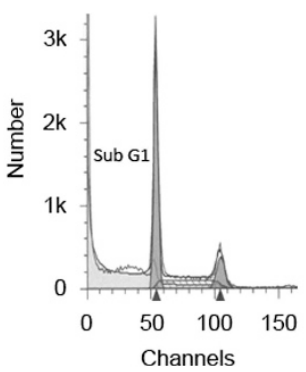

Flag-L5
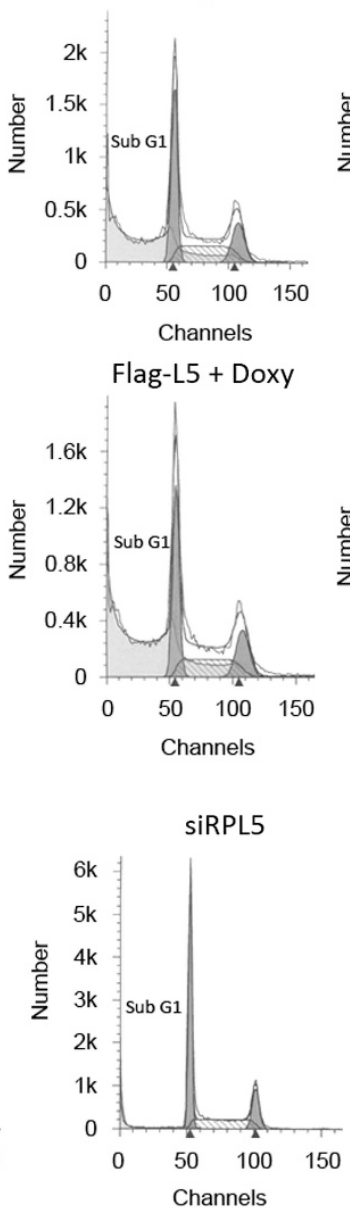

siRPL5 + Doxy

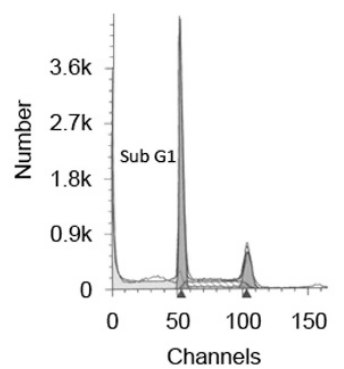

Flag-L11
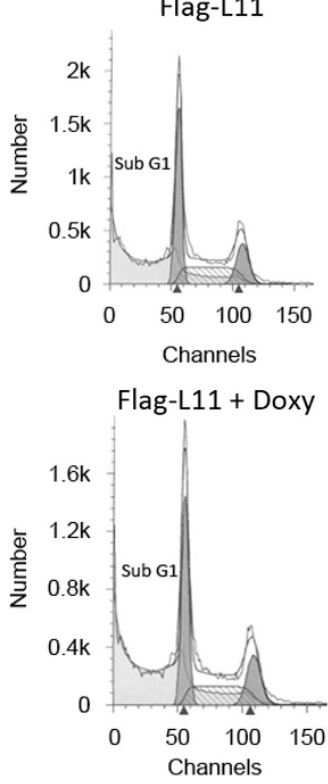

SiRPL11

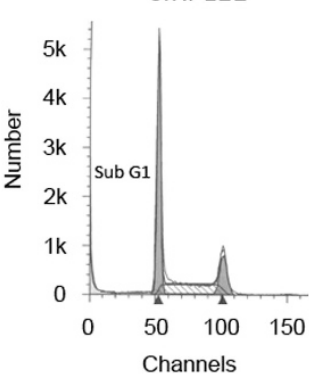

siRPL11 + Doxy

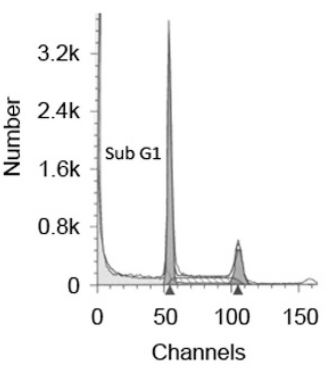

b

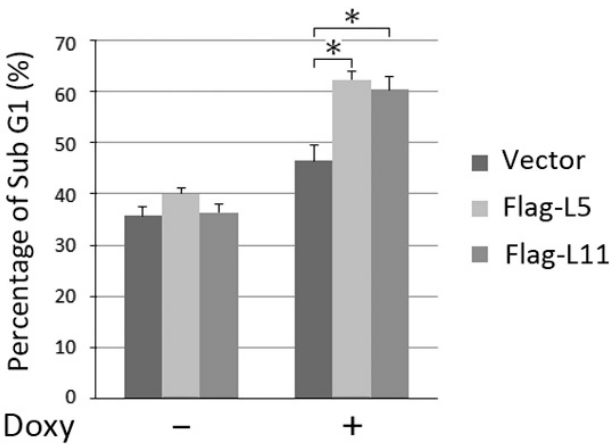

d

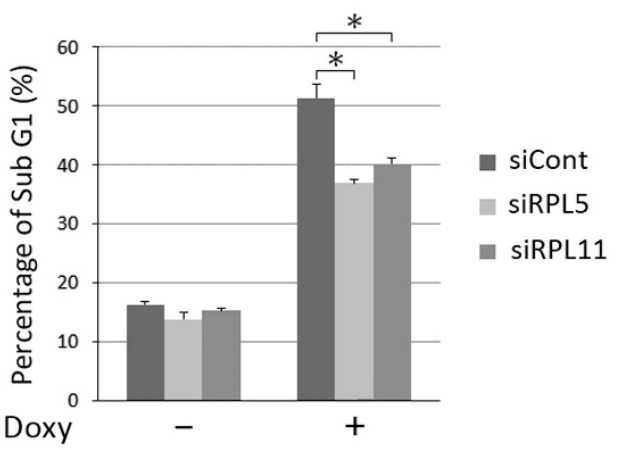

Figure 6 RPL5 and RPL11 enhance TAp73-induced apoptosis. (a, b) Ectopic expression of RPL5 and RPL11 prompt TAp73-mediated apoptosis in the Saos2 cells. Cells untreated or treated with Doxycycline were transfected with Flag-L5, Flag-L11 or vector as indicated and subjected to FACS analyses. The representative distribution of cell cycle and Sub-G1 is shown in (a); quantification of the Sub-G1 population is shown in (b). (*) $P$-value $\leq 0.05$. (c, d) Endogenous RPL5 and RPL11 are involved in TAp73-mediated apoptosis. Cells untreated or treated with Doxycycline were transfected with siRNAs against RPL5, RPL11 or control as indicated and subjected to FACS analyses. The representative distribution of cell cycle and Sub-G1 is shown in (c); quantification of the Sub-G1 population is shown in (d). (*) $P$-value $\leq 0.05$

Figure 5 Endogenous RPL5 and RPL11 regulate TAp73 activity. (a, b) Knockdown of RPL5 or RPL11 reduces the mRNA expression of TAp73 target genes, p21 (a) and Puma (b). HCT116 $6^{\text {p53-l- }}$ cells were transfected with siRNA against RPL5, RPL11 or control for $48-72 \mathrm{~h}$ and harvested for RT-qPCR analyses. $\left.{ }^{*}\right) P$-value $\leq 0.05$ versus siRNA control. (c) Knockdown of RPL5 or RPL11 reduces the protein levels of TAp73 target genes, p21, Puma and MDM2, as well as the level of C-PARP. The same experiments were executed as shown in (a) and (b), but the protein expression of p21, Puma, MDM2 and C-PARP was analyzed by IB using antibodies as indicated. (d) Ablation of RPL5 or RPL11 dampens TAp73-induced p21 and Puma expression. The TAp73-inducible Saos2 cells were transfected with indicated siRNAs and 5-FU and/or Doxycycline was supplemented before harvesting for IB analyses. (e) Reduction of Puma and Bax expression is TAp73 dependent. Cells were transfected with siRNAs against RPs and TAp73 as indicated, and treated with 5-FU before harvesting for RT-qPCR analyses. (f) Reduction of Puma and Bax expression is also through MDM2. The same experiments were performed as shown in (e) except that MDM2 siRNA was used instead 
a
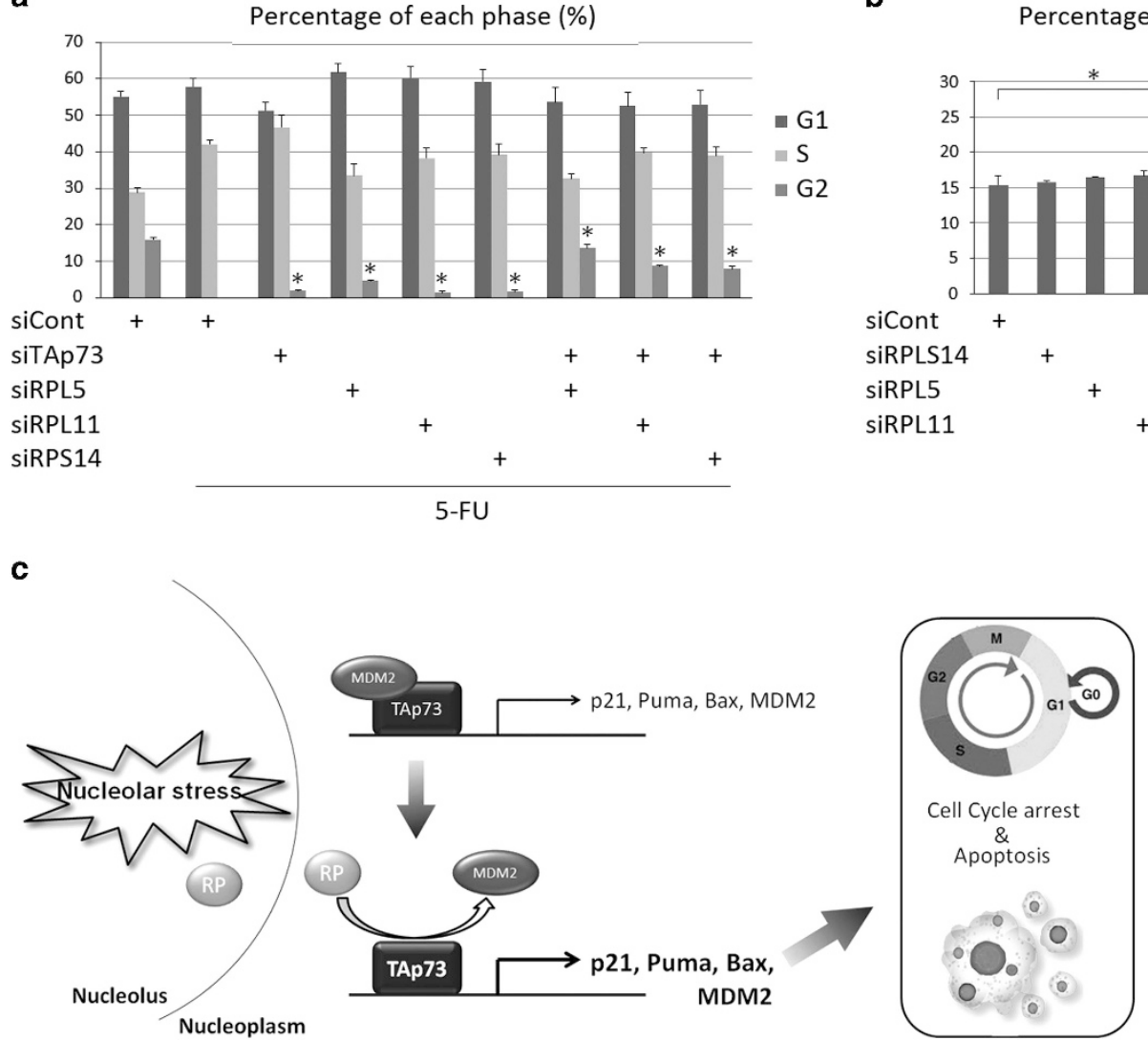

b

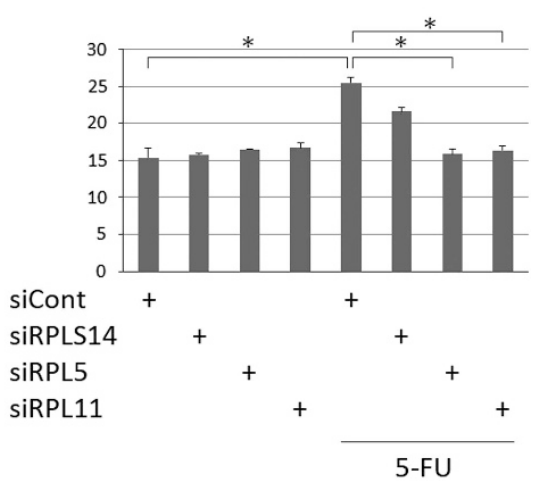

Figure 7 Both RPs and TAp73 are required for 5-FU-triggered S-phase cell cycle arrest and apoptosis. (a) RPL5/RPL11 and TAp73 have synergistic effects on arresting cells at S-phase in HCT116 ${ }^{\text {p53-I- }}$ cells in response to 5-FU treatment. Cells were transfected with combinations of siRNAs against RPL5, RPL11, TAp73 or control and treated with DMSO or 5-FU for $20 \mathrm{~h}$ as indicated before harvesting for FACS analyses. (*) $P$-value $\leq 0.05$ versus G2-phases of the sample 2. (b) Endogenous RPL5 and RPL11 are involved in 5 -FU-induced apoptosis in $\mathrm{HCT} 116^{\mathrm{p53}-l-}$ cells. Cells untreated or treated with 5-FU for $48 \mathrm{~h}$ were transfected with siRNAs against RPL5, RPL11 or control as indicated and subjected to FACS analyses. (*) $P$-value $\leq 0.05$. (c) A schematic diagram of the regulation of MDM2-TAp73 signaling by ribosomal proteins. TAp73 can transactivate $p 21$ and Puma, which are involved in cell growth inhibition, as well as MDM2, thus forming a feedback regulatory network as MDM2 may in turn suppress TAp73 activity. When cells were irritated by nucleolar stress (e.g., 5-FU treatment in this study), RPs would be prevented from the assembly of pre-ribosomes and released from the nucleolus to the nucleoplasm wherein they compromised the MDM2 function, resulting in TAp73 activation and consequent apoptosis and/or cell cycle arrest

the future. Since individual RPL5 or RPL11 molecules are able to activate TAp73, another remaining question would be if they cooperate with one another in this action. This appears to be possible, as these two RPs have been shown to synergistically activate p53 or inactivate c-Myc via $5 S$ rRNA. ${ }^{46,51}$ It will be tempting to determine if RPS14 might also work together with RPL5 and/or RPL11 in the near future. Our findings also provide a new molecular basis for the potential anti-cancer role for RPs, as TAp73 has been shown to be a tumor suppressor. ${ }^{2}$ Further interrogating this in a more physiological setting would offer more insight into how these RPs play roles in cancer development and cancer prevention. Because more RPs have been found to activate p53, but inactivate c-Myc, ${ }^{46,52-54}$ our findings together with others ${ }^{28,29}$ not only strengthen the notion that some RPs, such as RPL5, RPL11 and RPS14, possess intrinsic extra-ribosome functions in regulating other important cancer-relevant protein molecules, but also emphasize their potential roles as tumor suppressors.

\section{Materials and Methods}

Plasmids, primers and antibodies. The Flag-tagged RPL5, RPL11, RPS14, RPL30, RPS12, RPS19 and RPS27a, HA-MDM2, TAp73, GFP-TAp73, and
Myc- $\Delta$ Np73 mammalian expression plasmids and the His-tagged RPL5, RPL11 and MDM2, and GST-TAp73 plasmid for bacterial expression was described previously. ${ }^{15,18,26,27,52}$ The V5-tagged p53 plasmid was purchased from Addgene. Anti-RPL5, ${ }^{18}$ anti-RPL11, ${ }^{52}$ anti-HA (12CA5), anti-MDM2 (2A10 and 4B11) ${ }^{18,23}$ antibodies have been previously described. Anti-Flag (Sigma-Aldrich, St. Louis, MO, USA), anti-HA (Thermo Scientific, Waltham, MA, USA), anti-GFP (B-2, Santa Cruz Biotechnology, Santa Cruz, CA, USA), anti-Myc (9E10, Santa Cruz Biotechnology), anti-His (Thermo Scientific and Covance, Princeton, NJ, USA), anti-p73 (EP436Y, Epitomics, Burlingame, CA, USA), anti-p21 (CP74, NeoMarkers, Fremont, CA, USA), anti-Puma (H-136, Santa Cruz Biotechnology), anti-RPS14 (H-130, Santa Cruz Biotechnology) and anti-RPL30 (G-12, Santa Cruz Biotechnology) were commercially purchased.

Cell culture. Human H1299, HCT116 $6^{\mathrm{p} 53-/-}$ and MEF $\mathrm{P}^{\mathrm{p} 3-/- \text {;Mdm2-/- }}$ cells were cultured in Dulbecco's modified Eagle's medium supplemented with $10 \%$ fetal bovine serum, $50 \mathrm{U} / \mathrm{ml}$ penicillin and $0.1 \mathrm{mg} / \mathrm{ml}$ streptomycin at $37^{\circ} \mathrm{C}$ in a $5 \% \mathrm{CO} 2$ humidified atmosphere. Saos2 cell line for inducible expressed TAp73 was kindly provided by Dr. Vousden ${ }^{34}$ and maintained in the above mentioned condition.

Sucrose gradient fractionation and ribosome profiling. Cells treated with or without $5-\mathrm{FU}$ were harvested at $70-80 \%$ confluence after halting translation by $100 \mu \mathrm{g} / \mathrm{ml}$ cycloheximide. Cells were then lysed in lysis buffer $(10 \mathrm{mM}$ Tris- $\mathrm{HCl}(\mathrm{pH} 7.4), 5 \mathrm{mM} \mathrm{MgCl}_{2}, 100 \mathrm{mM} \mathrm{KCl}, 1 \%$ Triton X-100) and sheared with a 26 -gauge needle very gently. Lysates were subjected to $10-50 \%$ sucrose gradient 
centrifugation and the fractions were analyzed and collected through BR-188 Density Gradient Fractionation System (Brandel, Gaithersburg, MD, USA).

Transient transfection, immunoblot (IB) and immunoprecipitation (IP). Cells were transfected with plasmids as indicated in figure legends using TurboFect transfection reagent following the manufacturer's protocol (Thermo Scientific). Cells were harvested at 30-48 h post-transfection and lysed in lysis buffer consisting of $50 \mathrm{mM}$ Tris/ $\mathrm{HCl}(\mathrm{pH} 7.5), 0.5 \%$ Nonidet P-40 (NP-40), $1 \mathrm{mM}$ EDTA, $150 \mathrm{mM} \mathrm{NaCl}, 1 \mathrm{mM}$ phenylmethylsulfonyl fluoride, $1 \mathrm{mM}, 0.25 \mathrm{mg} / \mathrm{ml}$ pepstatin $A$ and $1 \mathrm{mM}$ leupeptin. Equal amounts of clear cell lysate $(20-50 \mathrm{mg})$ were used for IB analyses as described previously. ${ }^{26} \mathrm{IP}$ was conducted using antibodies as indicated in the figure legends and described previously. ${ }^{26}$ Beads were washed twice with lysis buffer, and once with RIPA buffer $(50 \mathrm{mM}$ Tris- $\mathrm{HCl}(\mathrm{pH}$ 8.0), $5 \mathrm{mM}$ EDTA, $1 \%$ NP-40, 0.5\% Deoxycholate, $0.1 \%$ SDS, $150 \mathrm{mM} \mathrm{NaCl}$ ). Bound proteins were detected by IB with antibodies as indicated in the figure legends.

ChIP. ChIP assay was performed using antibodies as indicated in the figure legends and described previously. ${ }^{55}$ The reverse cross-linked immuoprecipitated DNA fragments were purified using GeneJET gel extraction kit (Thermo Scientific) followed by qPCR analyses for the $p 21$ and Puma promoter using following primers: p21, 5'-GCTCCCTCATGGGCAAACTCACT-3' and 5-TGGCTGGTCTACCTGGCT CCTCT-3'; Puma, 5'-CTGTGGCCTTGTGTCTGTGAG-3' and 5'-CTAGCCCAAGG CAAGGAGGAC-3'.

Reverse transcription (RT) and quantitative PCR (qPCR) analyses. Total RNA was isolated from cells using Trizol (Invitrogen, Carlsbad, CA, USA) following the manufacturer's protocol. Total RNAs of $\sim 50 \mathrm{ng}$ were used as templates for reverse transcription using poly-(T)20 primers and M-MLV reverse transcriptase (Promega, Madison, WI, USA). Quantitative PCR (qPCR) was conducted using SYBR Green Mix according to the manufacturer's protocol (Bio-Rad, Hercules, CA, USA). Primers for p21, Puma and GAPDH cDNA detection are as follows: p21, 5'-CTGGACTGTTTTCTCTCGGCTC-3 and 5'-TGTATATTCAGC ATTGTGGGAGGA-3'; Puma, 5'-ACAGTACGAGCGGCGGAGACAA-3' and 5'-GGC GGGTGCAGGCACCTAATT-3'; Bax, 5'-CTGCAGAGGATGATTGCCGCCG-3' and 5'-TCCGGCACCTTGGTGCACAG-3'; GAPDH, 5'-GATTCCACCCATGGCAAATTC-3' and 5'-AGCATCGCCCCACTTGATT-3'.

GST fusion protein association assay. His-tagged MDM2, RPL5 and RPL11 proteins were expressed in E. coli, purified through a Ni-NTA (QIAGEN, Valencia, CA, USA) column, and eluted with $0.5 \mathrm{M}$ imidazole. Protein-protein interaction assays were conducted as described previously by using fusion proteincontaining glutathione beads. ${ }^{56}$ Briefly, $100 \mathrm{ng}$ of purified His-MDM2 protein were incubated and gently shaked with the glutathione-Sepharose 4B beads (SigmaAldrich) containing $500 \mathrm{ng}$ of GST-TAp73 or GST only at $4^{\circ} \mathrm{C}$ for $1 \mathrm{~h}$. A titrated amount of His-RPL5 or His-RPL11 was added to the reactions at the final concentration of $7 \mu \mathrm{M}$ and $14 \mu \mathrm{M}$. The mixtures were washed three times with GST lysis buffer (50 mM Tris/HCl pH 8.0, 0.5\% NP-40, 1 mM EDTA, $150 \mathrm{mM} \mathrm{NaCl}, 10 \%$ glycerol) after incubation for $1 \mathrm{~h}$ at $4^{\circ} \mathrm{C}$. Bound proteins were analyzed by IB with the antibodies as indicated in the figure legends.

Luciferase reporter assay. Cells were transfected with $\mathrm{pCMV}-\beta$-galactoside together with the plasmids as indicated in the figure legends. Luciferase activity was determined and normalized by a factor of $\beta$-Gal activity in the same assay as described previously. ${ }^{57}$

RNA interference. The siRNAs against RPL5, RPL11, TAp73 (Ambion, Carlsbad, CA, USA), RPS14 (Santa Cruz Biotechnology) and MDM2 (Life Technologies, Carlsbad, CA, USA) were commercially purchased. $10 \sim 100 \mathrm{nM}$ of siRNAs were introduced into cells using METAFECTENE siRNA transfection reagent following the manufacturer's protocol (Biontex Laboratories $\mathrm{GmbH}$, München, Germany). Cells were harvested 48 to $72 \mathrm{~h}$ after transfection for ChIP, immunoblot or qPCR. The Vector-based shRNAs of RPL5 and RPL11 were purchased (Sigma-Aldrich) and transfected using the standard transient transfection method described above.

Flow cytometry analyses (Fluorescence-activated cell sorting, FACS). Cells transfected with pcDNA, Flag-RPL5, Flag-RPL11, scrambled siRNA, RPL5 siRNA, RPL11 siRNA or RPS14 siRNA as indicated in the figure were fixed and stained in $500 \mathrm{ml}$ of propidium iodide (PI, Sigma-Aldrich) stain buffer ( $50 \mathrm{mg} / \mathrm{ml} \mathrm{PI}, 200 \mathrm{mg} / \mathrm{ml}$ RNase A, $0.1 \%$ Triton X-100 in phosphate-buffered saline) at $37^{\circ} \mathrm{C}$ for $30 \mathrm{~min}$. The cells were then analyzed for DNA content using a FACScan flow cytometer (BD Biosciences, San Jose, CA, USA). Data were analyzed using the CellQuest (BD Biosciences) and Modifit (Verity, Topsham, ME, USA) software programs.

Cell viability assay. To assess the long term cell growth, the Cell Counting Kit-8 (Dojindo Molecular Technologies, Rockville, MD, USA) was used according to the manufacturer's instructions. Cell suspensions were seeded at $5000 \mathrm{cells} /$ well with or without $2 \mu \mathrm{g} / \mathrm{ml}$ Doxy in 96-well culture plates at $12 \mathrm{~h}$ post-transfection. Cell-growth inhibition was determined by adding WST-8 at a final concentration of $10 \%$ to each well, and the absorbance of the samples was measured at $450 \mathrm{~nm}$ using a Microplate Reader (SpecrtraMax M5e, Molecular Device, Sunnyvale, CA, USA) every $24 \mathrm{~h}$ for 5 days.

\section{Conflict of Interest}

The authors declare no conflict of interest.

Acknowledgements. We thank Dr. Karen $\mathrm{H}$ Vousden for the TAp73-inducible cell line, Dr. Yi Sun for the RPS27a construct, and Mary Price for FACS analyses. HL was supported by $\mathrm{NIH-NCl}$ grants CA095441, CA $079721, \mathrm{CA} 129828$ and CA 172468.

1. Levine AJ, Oren M. The first 30 years of p53: growing ever more complex. Nat Rev Cancer 2009; 9: 749-758

2. Stiewe T. The p53 family in differentiation and tumorigenesis. Nat Rev Cancer 2007; 7: 165-168.

3. Oswald C, Stiewe T. In good times and bad: p73 in cancer. Cell Cycle 2008; 7: 1726-1731.

4. Tomasini R, Tsuchihara K, Wilhelm M, Fujitani M, Rufini A, Cheung CC et al. TAp73 knockout shows genomic instability with infertility and tumor suppressor functions. Genes Dev 2008; 22: 2677-2691.

5. Kaghad M, Bonnet H, Yang A, Creancier L, Biscan JC, Valent A et al. Monoallelically expressed gene related to $\mathrm{p} 53$ at $1 \mathrm{p} 36$, a region frequently deleted in neuroblastoma and other human cancers. Cell 1997; 90: 809-819.

6. Melino G, De Laurenzi V, Vousden KH. p73: friend or foe in tumorigenesis. Nat Rev Cancer 2002; 2: 605-615.

7. Jost CA, Marin MC, Kaelin WG Jr. p73 is a simian [correction of human] p53-related protein that can induce apoptosis. Nature 1997; 389: 191-194.

8. Wu X, Bayle JH, Olson D, Levine AJ. The p53-mdm-2 autoregulatory feedback loop. Genes Dev 1993; 7: 1126-1132.

9. Barak $Y$, Juven T, Haffner R, Oren M. mdm2 expression is induced by wild type p53 activity. EMBO J 1993; 12: 461-468.

10. Fuchs SY, Adler V, Buschmann T, Wu X, Ronai Z. Mdm2 association with p53 targets its ubiquitination. Oncogene 1998; 17: 2543-2547.

11. Kubbutat MH, Jones SN, Vousden KH. Regulation of p53 stability by Mdm2. Nature 1997; 387: 299-303.

12. Haupt $\mathrm{Y}$, Maya R, Kazaz A, Oren M. Mdm2 promotes the rapid degradation of $p 53$. Nature 1997; 387: 296-299.

13. Poyurovsky MV, Katz C, Laptenko O, Beckerman R, Lokshin M, Ahn J et al. The $\mathrm{C}$ terminus of $\mathrm{p} 53$ binds the N-terminal domain of MDM2. Nat Struct Mol Biol 2010; 17: 982-989.

14. Oliner JD, Pietenpol JA, Thiagalingam S, Gyuris J, Kinzler KW, Vogelstein B et al. Oncoprotein MDM2 conceals the activation domain of tumour suppressor p53. Nature 1993; 362: $857-860$.

15. Zeng $X$, Chen L, Jost CA, Maya R, Keller D, Wang $X$ et al. MDM2 suppresses p73 function without promoting p73 degradation. Mol Cell Biol 1999; 19: 3257-3266.

16. Dobbelstein M, Wienzek S, Konig C, Roth J. Inactivation of the p53-homologue p73 by the mdm2-oncoprotein. Oncogene 1999; 18: 2101-2106.

17. Balint $\mathrm{E}$, Bates $\mathrm{S}$, Vousden $\mathrm{KH}$. Mdm2 binds $\mathrm{p} 73$ alpha without targeting degradation. Oncogene 1999; 18: 3923-3929.

18. Dai MS, Lu H. Inhibition of MDM2-mediated p53 ubiquitination and degradation by ribosomal protein L5. J Biol Chem 2004; 279: 44475-44482.

19. Bai D, Zhang J, Xiao W, Zheng X. Regulation of the HDM2-p53 pathway by ribosomal protein L6 in response to ribosomal stress. Nucleic Acids Res 2014; 42: 1799-1811.

20. Zhang $Y$, Wolf GW, Bhat K, Jin A, Allio T, Burkhart WA et al. Ribosomal protein L11 negatively regulates oncoprotein MDM2 and mediates a p53-dependent ribosomal-stress checkpoint pathway. Mol Cell Biol 2003; 23: 8902-8912.

21. Lohrum MA, Ludwig RL, Kubbutat MH, Hanlon M, Vousden KH. Regulation of HDM2 activity by the ribosomal protein L11. Cancer Cell 2003; 3: 577-587.

22. Jin A, Itahana K, O'Keefe K, Zhang Y. Inhibition of HDM2 and activation of $\mathrm{p53}$ by ribosomal protein L23. Mol Cell Biol 2004; 24: 7669-7680. 
41. Li X, Amazit L, Long W, Lonard DM, Monaco JJ, O'Malley BW et al. Ubiquitin- and ATPindependent proteolytic turnover of p21 by the REGgamma-proteasome pathway. Mol Cell 2007; 26: 831-842.

42. Chen X, Barton LF, Chi Y, Clurman BE, Roberts JM. Ubiquitin-independent degradation of cell-cycle inhibitors by the REGgamma proteasome. Mol Cell 2007; 26: 843-852.

43. Sheaff RJ, Singer JD, Swanger J, Smitherman M, Roberts JM, Clurman BE et al. Proteasomal turnover of p21Cip1 does not require p21Cip1 ubiquitination. Mol Cell 2000; 5 : 403-410.

44. Chen X, Chi Y, Bloecher A, Aebersold R, Clurman BE, Roberts JM et al. N-acetylation and ubiquitin-independent proteasomal degradation of p21(Cip1). Mol Cell 2004; 16 : 839-847.

45. Lee H, Zeng SX, Lu H. UV Induces p21 rapid turnover independently of ubiquitin and Skp2. J Biol Chem 2006; 281: 26876-26883.

46. Liao JM, Zhou X, Gatignol A, Lu H. Ribosomal proteins L5 and L11 co-operatively inactivate c-Myc via RNA-induced silencing complex. Oncogene 2014; 33: 4916-4923.

47. Ongkeko WM, Wang XQ, Siu WY, Lau AW, Yamashita K, Harris AL et al. MDM2 and MDMX bind and stabilize the p53-related protein p73. Curr Biol 1999; 9: 829-832.

48. Zdzalik M, Pustelny K, Kedracka-Krok S, Huben K, Pecak A, Wladyka B et al. Interaction of regulators Mdm2 and Mdmx with transcription factors p53, p63 and p73. Cell Cycle 2010; 9: 4584-4591.

49. Zeng X, Li X, Miller A, Yuan Z, Yuan W, Kwok RP et al. The N-terminal domain of p73 interacts with the $\mathrm{CH} 1$ domain of $\mathrm{p} 300 / \mathrm{CREB}$ binding protein and mediates transcriptional activation and apoptosis. Mol Cell Biol 2000; 20: 1299-1310.

50. Gonzalez S, Prives C, Cordon-Cardo C. p73alpha regulation by Chk1 in response to DNA damage. Mol Cell Biol 2003; 23: 8161-8171.

51. Horn HF, Vousden KH. Cooperation between the ribosomal proteins $L 5$ and L11 in the p53 pathway. Oncogene 2008; 27: 5774-5784.

52. Dai MS, Arnold H, Sun XX, Sears R, Lu H. Inhibition of c-Myc activity by ribosomal protein L11. EMBO J 2007; 26: 3332-3345.

53. Challagundla KB, Sun XX, Zhang X, DeVine T, Zhang Q, Sears RC et al. Ribosomal protein $L 11$ recruits miR-24/miRISC to repress c-Myc expression in response to ribosomal stress. Mol Cell Biol 2011; 31: 4007-4021.

54. Chen MQ, Zhang AH, Zhang Q, Zhang BC, Nan J, Li X et al. Arabidopsis NMD3 is required for nuclear export of $60 S$ ribosomal subunits and affects secondary cell wall thickening. PLOS One 2012; 7: e35904.

55. Liao JM, Lu H. ChIP for identification of p53 responsive DNA promoters. Methods Mol Biol 2013; 962: 201-210.

56. Jin Y, Zeng SX, Dai MS, Yang XJ, Lu H. MDM2 inhibits PCAF (p300/CREB-binding protein-associated factor)-mediated p53 acetylation. I Biol Chem 2002; 277: 30838-30843.

57. Jin Y, Dai MS, Lu SZ, Xu Y, Luo Z, Zhao Y et al. 14-3-3gamma binds to MDMX that is phosphorylated by UV-activated Chk1, resulting in p53 activation. EMBO J 2006; 25: 1207-1218. 